\title{
ASYMPTOTIC PROPERTIES OF ENTIRE FUNCTIONS EXTREMAL FOR THE cos $\pi \rho$ THEOREM
}

\author{
BY DAVID DRASIN AND DANIEL F. SHEA ${ }^{1}$
}

Communicated by W. Fuchs, September 6, 1968

Let $f(z)$ be an entire function of order $\rho<1$. The classical "cos $\pi \rho$ theorem" of Valiron and Wiman [4, pp. 40,51] asserts that if

$$
\mu(r)=\min _{|z|=r}|f(z)|, \quad M(r)=\max _{|z|=r}|f(z)|,
$$

then, given $\epsilon>0$, the inequality

$$
\log \mu(r)>(\cos \pi \rho-\epsilon) \log M(r)
$$

holds for a sequence $r=r_{n} \rightarrow+\infty$.

We consider those functions $f(z)$ for which (1) is the best possible inequality, and discuss the global asymptotic behavior of such functions.

THEOREM 1. Let $f(z)$ be an entire function of order $\rho(0 \leqq \rho<1)$, and suppose

$$
\log \mu(r) \leqq[\cos \pi \rho+\epsilon(r)] \log M(r)
$$

where $\epsilon(r) \rightarrow 0$ as $r \rightarrow \infty$.

Then there exists a set $E$ of logarithmic density zero and a slowly varying function ${ }^{2} \psi(r)$ such that

$$
\begin{array}{rlrl} 
& \log M(r)=r^{\rho} \psi(r) & & (r \notin E), \\
n(r, 0)=[\sin \pi \rho / \pi+o(1)] r^{\rho} \psi(r) & (r \rightarrow \infty, r \in E)
\end{array}
$$

(where, as usual, $n(r, 0)$ denotes the number of zeros of $f(z)$ in $|z| \leqq r)$,

$$
\log \mu(r)=[\cos \pi \rho+o(1)] r^{p} \psi(r) \quad(r \rightarrow \infty, r \in E \cup H),
$$

where $H$ has (linear) density zero.

Further, there exists a real-valued function $\theta(r)$ such that if $k>1$ and $\delta>0$ are given and $\nu(r)$ denotes the number of zeros of $f(z)$ in the region

1 The first author was partially supported by NSF grant 4192-50-1395; the second author was partially supported by NSF grant GP-5728.

2 A function $\psi(r)$ is said to vary slowly if it is defined and positive for all $r>r_{0}$ and satisfies $\lim _{r \rightarrow \infty} \psi(\sigma r) / \psi(r) \rightarrow 1(0<\sigma<\infty)$. For a useful discussion of the properties of such functions see, for example, [9, p. 419]. For a discussion of linear and logarithmic densities see $[4$, p. 5]. 


$$
\left\{z: k^{-1} r \leqq|z| \leqq k r, \delta \leqq|\arg z-\theta(r)| \leqq \pi\right\},
$$

then

$$
\nu(r)=o\left(r^{p} \psi(r)\right) \quad(r \rightarrow \infty, r \notin E) .
$$

The function $\theta(r)$ oscillates slowly outside of $E$, in the sense that if $k>1$ and $\epsilon>0$ are given, then

$$
|\theta(t)-\theta(r)|<\epsilon \quad\left(r>r_{0}(\epsilon, k), r \notin E\right)
$$

holds for all $t$ in the interval $k^{-1} r \leqq t \leqq k r$.

The content of the conclusions (3)-(7) can be expressed more intuitively if we say that on almost all long intervals, $f(z)$ behaves like a Lindelöf function of order $\rho[12$, p. 18]. Indeed, it is not difficult to see that Theorem 1 implies that the asymptotic expansion

$$
\begin{aligned}
\log \left|f\left(t e^{i\left(\phi+\phi_{0}\right)}\right)\right|=[\cos \phi \rho+o(1)] \psi(r) t^{\rho} \\
\left(k^{-1} r \leqq t \leqq k r, t \in H,|\phi| \leqq \pi\right),
\end{aligned}
$$

is valid (uniformly in $t$ and $\phi$ ) as $r \rightarrow \infty$ outside $E$, where $\phi_{0}=\theta(r)-\pi$, $k>1$ is a given constant, and $H$ is the set of density zero given in Theorem 1.

Recent examples ${ }^{3}$ of W. K. Hayman [10] show that some exceptional set $E$ must be present in Theorem 1 ; when coupled with Theorem 2 below, they also show that even in the important special case when all the zeros of $f(z)$ are negative, $E$ cannot be replaced by a set of linear density 0 .

Theorem 1 may be compared to recent results of Kjellberg [11], Essén [7], Essén-Ganelius [8], and Anderson [1]. These authors consider (2) from another point of view; in particular, $\rho$ can be any number, $0<p<1$ (not necessarily the order of $f(z)$ ), but on the other hand, $\epsilon(r)$ must satisfy some condition such as

$$
\limsup _{R_{1}, R_{2} \rightarrow \infty} \int_{R_{1}}^{R_{2}} \frac{\epsilon(r) \log M(r)}{r^{1+\rho}} d r<M<\infty .
$$

Their conclusion, that $\log M(r) / r^{p}$ tends to a limit $\alpha(0 \leqq \alpha \leqq \infty)$ as $r \rightarrow \infty$ (with no need to avoid an exceptional set), is also of a different nature than that deduced here.

1. Outline of the proof. Let $f(z)$ satisfy the hypotheses of the theorem. We can assume that $f(0)=1$, and write

'Hayman's examples are valid only if $\rho=1 / 2$, but he comments that this case is probably typical. 


$$
f(z)=\prod_{n=1}^{\infty}\left(1-\frac{z}{a_{n}}\right), \quad F(z)=\prod_{n=1}^{\infty}\left(1+\frac{z}{\left|a_{n}\right|}\right)
$$

Since

$$
\log |F(-r)|+\log F(r) \leqq \log \mu(r)+\log M(r)
$$

$[4$, p. 40], it follows at once that $F(z)$ also satisfies the hypotheses of Theorem 1, and hence a theorem of P. D. Barry [3] yields that

$$
\log |F(-r)|=[\cos \pi \rho+o(1)] \log F(r) \quad\left(r \rightarrow \infty, r \in G^{*}\right),
$$

where $G^{*}$ has logarithmic density one. It is not hard to see, using (1.2) and hypothesis (2) again, that

$$
\log M(r) \sim \log F(r) \quad\left(r \rightarrow \infty, r \in G^{*}\right) .
$$

An easy extension of Theorem 2 of [2] now shows that from (1.3) follows

$$
\nu(r)=o(\log M(r)) \quad\left(r \rightarrow \infty, r \in G^{*}\right) .
$$

We next establish that $G^{*}$ can be replaced by a subset $G_{*}$ having the following crucial properties: there are sequences $\left\{\alpha_{n}\right\},\left\{\beta_{n}\right\}$ and a set $H$ of (linear) density zero such that

$$
G_{*}=\bigcup_{n=1}^{\infty}\left[\alpha_{n}, \beta_{n}\right]-H \quad\left(\alpha_{n} \rightarrow \infty, \beta_{n} / \alpha_{n} \rightarrow \infty\right)
$$

has logarithmic density one, and, if $k>1$ is given, then

$$
\left[k^{-1} \alpha_{n}, k \beta_{n}\right] \subset G^{*} \cup H \quad\left(n>n_{0}(k)\right) .
$$

The exceptional set $E$ which appears in the statement of Theorem 1 is the complement of $G=\bigcup_{n=1}^{\infty}\left[\alpha_{n}, \beta_{n}\right]$.

In view of elementary properties of sets of linear density zero, it is easy to see that (1.3) holds with $G^{*}$ replaced by $G$, and so it suffices to prove (3)-(5) for $F(z)$. The argument hinges now on a suitable generalization (to allow exceptional sets of logarithmic density zero) of the following theorem [6], which is one form of a complement to some classical results of Titchmarsh [13] and Bowen and Macintyre [5].

THEOREM 2. Let $F(z)$ be an entire function of the form (1.1), and suppose

$$
\frac{\log |F(-r)|}{\log F(r)} \rightarrow \alpha \quad(r \rightarrow \infty, r \in H),
$$

where $H$ is of (linear) density zero. 


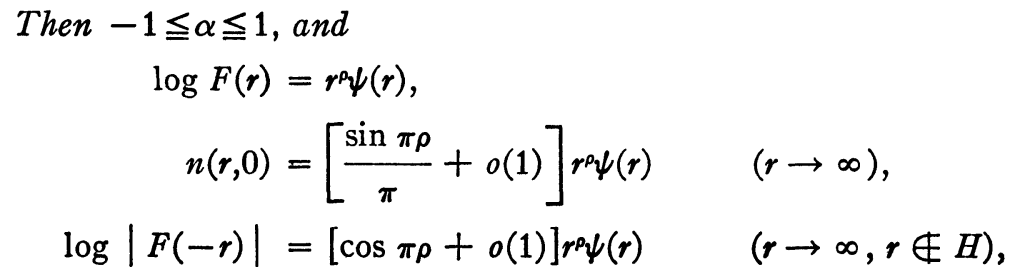

where $\rho$ is determined by

$$
\cos \pi \rho=\alpha \quad(0 \leqq \rho \leqq 1),
$$

and $\psi$ is a slowly varying function.

Finally, (6) follows from (1.4) and (3), and (7) is an easy consequence of (6) (cf. [2, Corollary 1]).

Conclusion (3) of Theorem 1 implies that $f(z)$ has regular growth in the sense of Valiron. Analogues of Theorem 1, valid for functions of irregular growth, can also be derived from these methods.

\section{REFERENCES}

1. J. M. Anderson, Asymptotic properties of integral functions of genus zero, Quart. J. Math. Oxford Ser. (2) 16 (1965), 151-165.

2. - The behaviour of integral and subharmonic functions, J. Analyse Math. 17 (1966), 185-208.

3. P. D. Barry, Some theorems related to the cos $\pi \rho$ theorem, A. J. Macintyre memorial volume, Ohio Univ. Press (to appear).

4. R. P. Boas, Entire functions, Academic Press, New York, 1954.

5. N. A. Bowen and A. J. Macintyre, Some theorems on integral functions with negative zeros, Trans. Amer. Math. Soc. 70 (1951), 114-126.

6. D. Drasin and D. F. Shea, Complements to some theorems of Bowen and Macintyre on the radial growth of entire functions with negative zeros, A. J. Macintyre memorial volume, Ohio Univ. Press, (to appear).

7. M. Essén, $A$ theorem on the maximum modulus of entire functions, Math. Scand. 17 (1965), 161-168.

8. M. Essén and T. Ganelius, Asymptotic properties of entire functions of order less than one, Proc. Sympos. Pure Math., vol. 11, Amer. Math. Soc., Providence, R. I., 1968.

9. W. Feller, Introduction to probability theory and its applications. Vol. II, Wiley, New York, 1966.

10. W. K. Hayman, Some examples related to the cos $\pi \rho$-theorem (to appear).

11. B. Kjellberg, $A$ theorem on the minimum modulus of entire functions, Math. Scand. 12 (1963), 5-11.

12. R. Nevanlinna, Le théorème de Picard-Borel et la théorie des fonctions méromorphes, Gauthier-Villars, Paris, 1929.

13. E. C. Titchmarsh, On integral functions with real negative zeros, Proc. London Math. Soc. (2) 26 (1927), 185-200.

Purdue University, Lafayette, Indiana 47907 and

UNIVERSITY OF Wisconsin, Madison, Wisconsin 53706 